\title{
Multi-level Graph Drawing using Infomap Clustering *
}

\author{
Seok-Hee Hong ${ }^{1}$, Peter Eades ${ }^{1}$, Marnijati Torkel ${ }^{1}$, Ziyang Wang ${ }^{1}$, \\ David Chae ${ }^{1}$, Sungpack Hong ${ }^{2}$, Daniel Langerenken ${ }^{2}$, and Hassan Chafi ${ }^{2}$ \\ 1 University of Sydney, Australia \\ \{seokhee.hong, peter . eades, mtor0581, zwan0130, min. chae\} @sydney . edu . au \\ 2 Oracle Research lab, US \\ \{sungpack.hong, daniel.langerenken, hassan.chafi\}@oracle.com
}

\begin{abstract}
Infomap clustering finds the community structures that minimize the expected description length of a random walk trajectory; algorithms for infomap clustering run fast in practice for large graphs. In this paper we leverage the effectiveness of Infomap clustering combined with the multi-level graph drawing paradigm. Experiments show that our new Infomap based multi-level algorithm produces good visualization of large and complex networks, with significant improvement in quality metrics.
\end{abstract}

\section{Introduction}

The multi-level graph drawing is a popular approach to visualize large and complex graphs to improve the quality of drawings. It recursively coarsens the graph and then uncoarsens the drawing using layout refinement. There are a number of multi-level graph drawing algorithms available [7/9|11|14|17|18|20]. They mainly differ in the coarsening method.

Clustering is a widely used analysis method for identifying groups with strong similarity, or communities in the data. Graph clustering is to partition a graph such that vertices in the same cluster are more interconnected. Infomap clustering computes clusters by translating a graph into a map, which decomposes the myriad nodes and links into modules that represent the graph [19]. It maximizes an objective function called the minimum description length of a random walk trajectory, where the approximation to the optimal solution can be computed quickly. Infomap performed the best in community finding experiments [15.

In this paper, we present a new multi-level graph drawing algorithm based on Infomap clustering. More specifically, we leverage the effectiveness of Infomap clustering, combined with the multi-level graph drawing paradigm. Experiments with real-world large and complex networks such as protein-protein interaction networks, Facebook graph, Autonomous Systems (AS) graphs as well as benchmark graphs show that our new multi-level algorithms produce good visualization with significant improvement in quality metrics, including shape-based metrics [4, edge crossing and stress. It also requires a small number of coarsening steps for medium to large graphs, which makes it fast to run.

\footnotetext{
* Research supported by ARC Linkage Grant with Oracle labs.
} 


\section{Related Work}

Hadany and Harel presented the multi-scale method using an edge contraction based coarsening method and a force-directed layout preserving topological properties such as cluster size and vertex degree 10. Koren and Harel presented FMS, which used a $k$-center approximation based coarsening method and a forcedirected layout with a beautification [14.

Walshaw presented a multi-level algorithm using a matching, by repeatedly collapsing maximal independent subsets of graph edges, and a grid variant of Fruchterman-Reingold [6] layout [20. Gajer et al. presented GRIP using a maximum independent set filtration based coarsening method, and an intelligent initial placement of vertices based on both graph and Euclidean distances [7.

Quigley and Eades presented FADE using the quad tree, and Barnes-Hut $n$ body method 1 for approximation of the repulsive force computation in a forcedirected layout 18 . Hachul and Junger presented $\mathrm{FM}^{3}$ using similar method to compute the repulsive forces between vertices, where subgraphs with small diameter, called solar system, are partitioned and collapsed to obtain a multilevel representation [9. Hu presented the $s f d p$ layout, also using the Barnes-Hut approximation method [11]. Frishman and Tal [5] presented a multi-level force directed graph layout on the GPU, based on spectral partitioning and KamadaKawai layout [12. Bartel et al. presented an experimental study for extensive comparison of various multi-level algorithms, using a combination of coarsening methods, initial placement and graph layout methods [2].

More recently, Meyerhenke et al. presented a multi-level algorithm using a label propagation method for the coarsening step, and Maxent stress optimisation layout [8] on shared memory parallelization [16. Nguyen and Hong used fast $k$-core coarsening method, which can be computed in linear time [17.

\section{Infomap based Multi-level Algorithm}

The multi-level graph drawing algorithm is an iterative process consisting of the following three steps: coarsening, initialization (or placement), and graph layout (or refinement). Roughly speaking, the coarsening step is to cluster vertices to define a smaller graph, recursively until the size of the graph falls below the threshold, resulting in a coarse graph hierarchy, $G_{0}, G_{1}, \ldots, G_{L}$. The layout of graph $G_{L}$ is then extended to the layout of graph $G_{L-1}$ by placement (i.e., add vertices back to the layout) and refinement step. Recursively, these steps extend the layout of graph $G_{L}$ to $G_{0}$ by repetitively interpolating from $G_{i}$ to $G_{i-1}$. In each iteration, the layout of $G_{i}$ is used to compute an initial placement of $G_{i-1}$, and then the layout algorithm is applied to refine the layout.

\subsection{Coarsening: Infomap Clustering}

Let $G=(V, E)$ be a graph with vertex set $V$ and edge set $E$. The coarsening step computes a graph level hierarchy by iteratively computing a sequence of smaller graphs $G_{0}, G_{1}, G_{2}, \ldots, G_{L}$, where the original graph $G=G_{0}$. At each level, a coarser graph (or clustered graph) is computed by combining a sets of 
vertices belong to the same cluster in $G_{i}$ and replacing into a single vertex in $G_{i+1}$, recursively until the predefined stop criterion is satisfied.

Infomap clustering finds community structure that minimizes the expected description length of a random walk trajectory [19. It computes clusters by translating a graph into a map, which decomposes the myriad nodes and links into modules that represent the graph. The algorithm maximizes an objective function called the Minimum Description Length.

We first compute the Infomap clustering of $G$, and partition the vertex set $V$ into $V_{i}$ based on the clusters. More specifically, we define a clustered graph with a weighted vertex set (i.e., the number of vertices belong to each cluster) and a weighted edge set (i.e., the number of edges between the partitioned vertex set). The vertices $u_{1}, u_{2}, \ldots, u_{k} \in V_{i}$ are merged to form a new cluster vertex $v \in V_{i+1}$, where the weight of $v$ is computed as $|v|=\left|u_{1}\right|+\left|u_{2}\right|+\ldots+\left|u_{k}\right|$. Similarly, the weight of the collapsed edges are computed as the sum of the weights of the edges that it replaces. This coarsening phase stops when the resulting clustered graph has a small size (say 50) or there is no reduction in terms of size.

\subsection{Initialization: Placement}

This step aims to compute a good initial layout of $G_{i-1}$ using the layout of $G_{i}$. Let $v_{i} \in V_{i}$ of $G_{i}$ corresponds to a cluster of vertices $u_{1}, u_{2}, \ldots, u_{k} \in V_{i-1}$ of $G_{i-1}$. We add back vertices $u_{j}, j=1, \ldots, k$ to the layout of $G_{i}$ by initializing the positions of $u_{j}$ using the position of $v_{i}$. Here we use the following three variations.

- Circle placement: It places all $u_{j}, j=1, \ldots, k$ at the circle with a small radius, where the center of the circle is the location of $v_{i}$.

- Barycenter placement: It places each vertex at the barycenter of its neighbors [7].

- Zero placement: It places all $u_{j}, j=1, \ldots, k$ at the same position as $v_{i}$ with small perturbation [20].

\subsection{Refinement: Force-directed Layout}

The initial layout of $G_{i-1}$ is recursively refined at each level using a forcedirected algorithm. We use layout algorithms, previously used in other multi-level graph drawing algorithm experiments [2]:

- FR: Fruchterman and Reingold layout [6].

- FRG: grid variant of Fruchterman and Reingold layout, used in [20].

- FME (Fast-Multipole Embedder): an improvement of NME (New Multipole Method) layout of $\mathrm{FM}^{3}[9$, designed for a multi-level method in [2].

\section{Experiments}

We implemented Infomap clustering based multi-level algorithm using OGDF [3], which was used in the comparison experiments of multi-level algorithms [2]. We used a standard Dell laptop with Intel Core i7, 16 GB RAM.

We first experimented with three different placement methods, and found that there is no significant difference in terms of layout quality. We choose the 
barycenter placement, which shows slightly better performance, with three layouts FR, FRG and FME for comparison.

More specifically, we have the following variations for comparison:

- InfomapFR: Infomap multi-level algorithm with FR layout

- InfomapFRG: Infomap multi-level algorithm with FR grid variant layout

- InfomapFME: Infomap multi-level algorithm with FME layout

The experiment was conducted with real-world benchmark data sets including social networks such as facebook, biological networks such as protein-protein interaction networks, and benchmark graphs used in previous work 2/17/20.

Table 1 shows the details of the data sets, the number of coarsening levels and runtime (seconds), where $D$ represents the density of a graph $G$ and $L$ represents the number of levels. We can clearly see that the Infomap coarsening method produced small number of levels such as 2 or 3 for most of data sets. Overall, Infomap clustering runs quite fast for medium size graphs.

\begin{tabular}{|l|r|r|r|r|r|r|r|r|r||r||r|}
\hline Graph $G$ & $\left|V_{0}\right|$ & $\left|E_{0}\right|$ & $D$ & $L$ & Time & $\left|V_{1}\right|$ & $\left|E_{1}\right|$ & $\left|V_{2}\right|$ & $\left|E_{2}\right|$ & $\left|V_{3}\right|$ & $\left|E_{3}\right|$ \\
\hline$G_{-} 15 \_0$ & 1785 & 20459 & 11.5 & 2 & 0.02 & 59 & 100 & 9 & 8 & & \\
\hline nasa1824 & 1824 & 18692 & 10.3 & 2 & 0.02 & 53 & 217 & 5 & 7 & & \\
\hline$G_{-}$-0 & 2075 & 4769 & 2.3 & 2 & 0.02 & 89 & 326 & 8 & 11 & & \\
\hline yeastppi & 2361 & 7182 & 3.0 & 2 & 0.04 & 302 & 1923 & 101 & 0 & & \\
\hline soc_h & 2426 & 11630 & 4.8 & 2 & 0.02 & 301 & 1088 & 149 & 1 & & \\
\hline oflights & 2939 & 15677 & 5.3 & 2 & 0.03 & 170 & 477 & 19 & 24 & & \\
\hline ecolippi & 3796 & 78120 & 20.6 & 2 & 0.03 & 245 & 2453 & 53 & 1 & & \\
\hline facebook & 4039 & 88234 & 21.9 & 2 & 0.02 & 93 & 272 & 7 & 11 & & \\
\hline 3elt & 4720 & 13722 & 2.9 & 2 & 0.05 & 189 & 489 & 17 & 35 & & \\
\hline USpowerGrid & 4941 & 6594 & 1.3 & 2 & 0.18 & 489 & 963 & 44 & 104 & & \\
\hline as19990606 & 5188 & 10974 & 2.1 & 2 & 0.17 & 368 & 2034 & 12 & 38 & & \\
\hline commanche_dual & 7920 & 19800 & 2.5 & 2 & 0.24 & 503 & 1365 & 34 & 71 & & \\
\hline p2p-Gnutella05 & 8846 & 31839 & 3.6 & 2 & 0.20 & 830 & 18154 & 3 & 0 & & \\
\hline astroph2001 & 16046 & 121251 & 7.6 & 3 & 0.61 & 1219 & 9333 & 395 & 68 & 369 & 0 \\
\hline condmat2001 & 16264 & 47594 & 2.9 & 3 & 1.33 & 1720 & 4574 & 798 & 774 & 726 & 0 \\
\hline crack-dual & 20141 & 30043 & 1.5 & 3 & 1.16 & 1357 & 3633 & 84 & 216 & 10 & 18 \\
\hline bcsstk31 & 35588 & 608502 & 17.1 & 2 & 0.36 & 453 & 2295 & 25 & 44 & & \\
\hline shock-9 & 36476 & 71290 & 2.0 & 3 & 1.17 & 1351 & 3852 & 74 & 191 & 8 & 14 \\
\hline del16 & 65536 & 196575 & 3.0 & 3 & 1.95 & 1981 & 5921 & 101 & 290 & 8 & 16 \\
\hline
\end{tabular}

Table 1. Data sets, size, number of levels $(L)$ and runtime.

Comparison of Quality Metrics: For large and complex graphs, edge crossing may not a suitable metric to measure the quality of drawings [413. We used the shape-based metrics [4]; this is a new graph drawing quality measure specially designed for large graphs. Roughly speaking, the shape-based metrics measure the faithfulness of graph drawing, i.e., how well the shape of the drawing represents the structure (or shape) of the graph. 


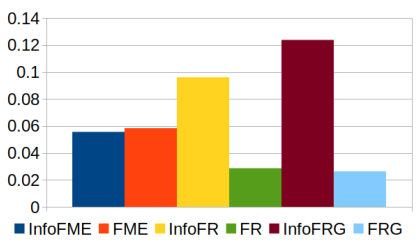

(a) Shape-based (larger, better)

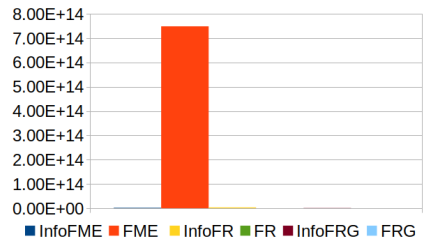

(b) stress (smaller, better)

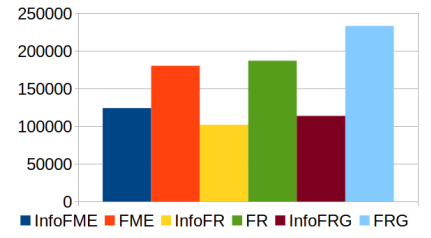

(c) crossing (smaller, better)

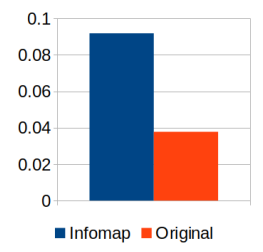

(d) Shape-based

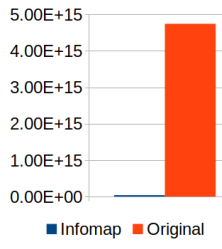

(e) stress

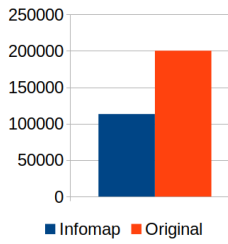

(f) crossing
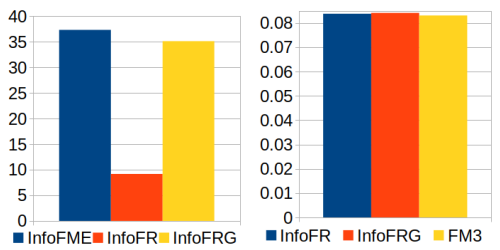

(g) Improvement

(h) Infomap vs. $F M^{3}$

Fig. 1. Significant improvement in quality metrics: (a)(b)(c) Average of metrics per layout: InfomapFME (blue), FME (red), InfomapFR (yellow), FR (green), InfomapFRG (brown), FRG (cyan); (d)(e)(f) Average of metrics: Infomap (blue) vs. Original (red); (g) Average of improvement by Infomap over Original in Shape-based metrics: InfomapFME (blue), InfomapFR (red), InfomapFRG (yellow); (h) Average of shapebased metrics: InfomapFR (blue), InfomapFRG (red), $F M^{3}$ (yellow).

Figures 1(a), (b) and (c) show the comparison of average metrics between six layouts (i.e., Infomap multi-level vs. FME, FRG, FR original layouts) using shape-based quality metrics $(Q)$, stress and edge crossings. Clearly, we can see that Infomap multi-level layouts perform significantly better than the original layouts. In general, InfomapFR and InfomapFRG perform better than InfomapFME. Figures 1(d), (e) and (f) show the average metrics between Infomap multi-level and original layouts. Overall, we can see that Infomap multi-level layouts outperform original layouts. Figure $1(\mathrm{~g})$ shows the average improvement by Infomap multi-level layouts over original layouts in shape-based metrics (i.e., $\left.\left(Q_{\text {Infomap }} / Q_{\text {Original }}-1\right)\right)$. Clearly, significant improvement was achieved by InfomapFME and InfomapFRG.

Visual Comparison: Overall, Infomap multi-level layouts perform significantly better than original layouts. In general, InfomapFR and InfomapFRG perform significantly better than other layouts, and InfomapFME achieved the most significant improvement over FME. For example, Figure 2 shows visual comparison between layouts of 3elt.

Comparison with $F M^{3}$ : Figure 1(h) shows average shape-based metrics between InfomapFR, InfomapFRG and $F M^{3}$, excluding the outlier. Clearly, we can see that InfomapFR and Infomap FRG perform similar to $F M^{3}$ in shape- 
based metrics. For layout comparison, see Figures 3 . We can see that InfomapFR perform similar to $F M^{3}$, and for some instances perform better than $F M^{3}$.

Summary: Our experimental results provide strong evidence that our Infomap based multi-level algorithm performs considerably well for real-world social networks, biological networks and benchmark graphs.

- Overall, Infomap multi-level layouts perform significantly better than original layouts in terms of quality metrics and visualisation.

- Metric wise, InfomapFR and InfomapFRG perform better than InfomapFME.

- InfomapFME achieved the most significant improvement.

- InfomapFR and InfomapFRG perform similar to $F M^{3}$ in terms of shapebased metrics and visual comparison.

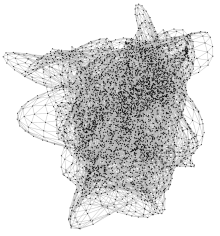

(a) FME

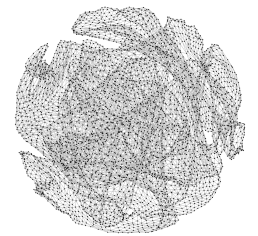

(d) Infomap FME

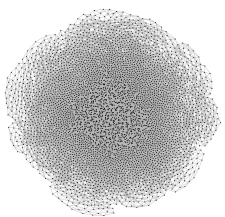

(b) FRG

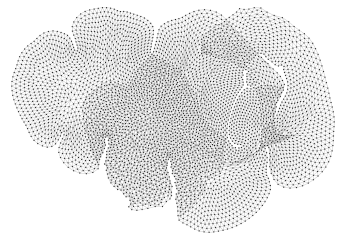

(e) Infomap FRG

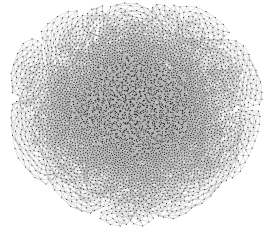

(c) FR

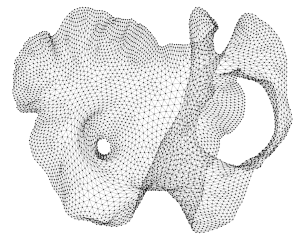

(f) Infomap FR

Fig. 2. Visual comparison of 3elt.

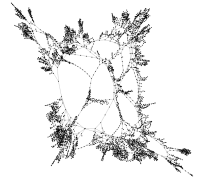

(a) $F M^{3}$

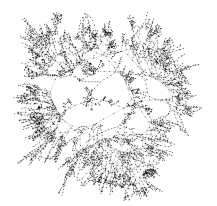

(b) Infomap FR

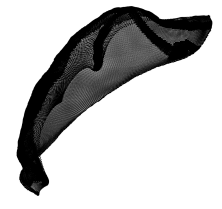

(c) $F M^{3}$

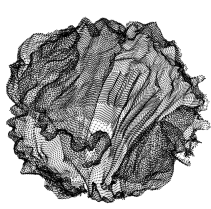

(d) Infomap FR

Fig. 3. Comparison with $F M^{3}$ : (a)(b)USpowerGrid; (c)(d) shock-9 


\section{References}

1. Barnes, J., Hut, P.: A hierarchical o $(n \log n)$ force-calculation algorithm. Nature 324 (1986)

2. Bartel, G., Gutwenger, C., Klein, K., Mutzel, P.: An experimental evaluation of multilevel layout methods. In: Graph Drawing - 18th International Symposium, GD 2010, Konstanz, Germany, September 21-24, 2010. Revised Selected Papers. pp. 80-91 (2010)

3. Chimani, M., Gutwenger, C., Jünger, M., Klau, G.W., Klein, K., Mutzel, P.: The open graph drawing framework (OGDF). In: Handbook on Graph Drawing and Visualization., pp. 543-569 (2013)

4. Eades, P., Hong, S., Klein, K., Nguyen, A.: Shape-based quality metrics for large graph visualization. In: Graph Drawing and Network Visualization - 23rd International Symposium, GD 2015. pp. 502-514 (2015)

5. Frishman, Y., Tal, A.: Multi-level graph layout on the GPU. IEEE Trans. Vis. Comput. Graph. 13(6), 1310-1319 (2007)

6. Fruchterman, T.M., Reingold, E.M.: Graph drawing by force-directed placement. Software: Practice and experience 21(11), 1129-1164 (1991)

7. Gajer, P., Kobourov, S.G.: Grip: Graph drawing with intelligent placement. J. Graph Algorithms Appl. 6(3), 203-224 (2002)

8. Gansner, E.R., Hu, Y., North, S.C.: A maxent-stress model for graph layout. IEEE Trans. Vis. Comput. Graph. 19(6), 927-940 (2013)

9. Hachul, S., Jünger, M.: Drawing large graphs with a potential-field-based multilevel algorithm. In: Graph Drawing. pp. 285-295. Springer (2005)

10. Hadany, R., Harel, D.: A multi-scale algorithm for drawing graphs nicely. Discrete Applied Mathematics 113(1), 3-21 (2001)

11. $\mathrm{Hu}, \mathrm{Y} .:$ Efficient, high-quality force-directed graph drawing. Mathematica Journal 10(1), 37-71 (2005)

12. Kamada, T., Kawai, S.: An algorithm for drawing general undirected graphs. Information processing letters 31(1), 7-15 (1989)

13. Kobourov, S.G., Pupyrev, S., Saket, B.: Are crossings important for drawing large graphs? In: Graph Drawing - 22nd International Symposium, GD 2014, Würzburg, Germany, September 24-26, 2014, Revised Selected Papers. pp. 234-245 (2014)

14. Koren, D., Harel, Y.: A fast multi-scale method for drawing large graphs. Journal of graph algorithms and applications 6(3) (2002)

15. Lancichinetti, A., Fortunato, S.: Community detection algorithms: A comparative analysis. Phys. Rev. E 80, 056117 (Nov 2009)

16. Meyerhenke, H., Nöllenburg, M., Schulz, C.: Drawing large graphs by multilevel maxent-stress optimization. In: Graph Drawing and Network Visualization - 23rd International Symposium, GD 2015. pp. 30-43 (2015)

17. Nguyen, A., Hong, S.: k-core based multi-level graph visualization for scale-free networks. In: 2017 IEEE Pacific Visualization Symposium, PacificVis 2017, Seoul, South Korea, April 18-21, 2017. pp. 21-25 (2017)

18. Quigley, A., Eades, P.: Fade: Graph drawing, clustering, and visual abstraction. In: Graph Drawing. pp. 197-210. Springer (2001)

19. Rosvall, M., Bergstrom, C.T.: Maps of random walks on complex networks reveal community structure. Proceedings of the National Academy of Sciences 105(4), 1118-1123 (2008)

20. Walshaw, C., et al.: A multilevel algorithm for force-directed graph-drawing. J. Graph Algorithms Appl. 7(3), 253-285 (2003) 